INFN-ISS 94/3

March 94

\title{
Hard constituent quarks and electroweak properties of pseudoscalar mesons
}

\author{
F. Cardarelli ${ }^{(a)}$, I.L. Grach $^{(b)}$, I.M. Narodetskii ${ }^{(b)}$ \\ E. Pace ${ }^{(a)}$, G. Salmè ${ }^{(c)}$, S. Simula ${ }^{(c)}$ \\ ${ }^{(a)}$ Department of Physics, University of Rome "Tor Vergata" \\ and INFN Sezione Tor Vergata \\ Via della Ricerca Scientifica, I-00133 Roma, Italy \\ ${ }^{(b)}$ Institute of Theoretical and Experimental Physics \\ Moscow 117259, Russia \\ (c) Istituto Nazionale di Fisica Nucleare, Sezione Sanità, \\ Viale Regina Elena 299, I-00161 Roma, Italy
}

\begin{abstract}
The high momentum components generated in the wave function of pseudoscalar mesons by the one-gluon-exchange interaction are investigated within a relativistic constituent quark model. Adopting the light-cone formalism, the sensitivity of the weak decay constant and the charge form factor to hard constituent quarks is illustrated.
\end{abstract}

Phys. Lett. B, in press. 
The investigation of the electroweak properties of mesons and baryons can shed light on the effective constituents of hadrons and their dynamics. In particular, the understanding of hadron electroweak properties has recently received much theoretical attention within the context of constituent quark models both in the framework of light-cone dynamics [1, 2, 3, 4] and in a diagrammatic approach [5]. It has also been argued that existing experimental data on the weak decay constant and the charge form factor of mesons can be accounted for within a constituent $q \bar{q}$ picture 44. However, such a conclusion has been obtained by adopting a simplified description of the dynamics of the constituent quarks inside the meson, namely, by using gaussian-like wave functions which are expected to describe the effects of the confinement scale only. In this letter the wave functions of pseudoscalar (S-wave) mesons are analyzed within the relativized constituent quark model of ref. [6], which properly describes meson (as well as baryon [7]) mass spectra in terms of an effective (QCD motivated) $q \bar{q}$ interaction composed by a one-gluon-exchange term and a linear confining potential. It is shown that high momentum components, generated in the meson wave function by the onegluon-exchange term, sharply affect the weak decay constant and the charge form factor. The agreement with existing experimental data can be obtained by introducing an axial-vector coupling constant and a charge form factor of the constituent quark. As in refs. [1, 2, 3, 4], meson electroweak properties will be evaluated adopting the light-cone formalism [8], which represents the natural framework for constructing a relativistic quark model featuring the dominance of the $q \bar{q}$ component of mesons [9].

1. Light-cone meson wave functions. As is known, light-cone wave functions are eigenfunctions of the mass operator, e.g.

$$
M=M_{0}+V
$$

and of the usual angular momentum operators $j^{2}$ and $j_{n}$, where the vector $\hat{\mathbf{n}}=(0,0,1)$ defines the spin quantization axis. In eq. (1) $V$ is a Poincaré invariant interaction term and $M_{0}$ is the free mass operator, which reads as

$$
M_{0}^{2}=\frac{k_{\perp}^{2}+m_{q}^{2}}{\xi}+\frac{k_{\perp}^{2}+m_{\bar{q}}^{2}}{1-\xi}
$$

where $m_{q}\left(m_{\bar{q}}\right)$ is the constituent quark (antiquark) mass and the intrinsic light-cone variables $\vec{k}_{\perp}$ and $\xi$ are

$$
\begin{array}{r}
\xi=p_{q}^{+} / P^{+}=1-p_{\bar{q}}^{+} / P^{+} \\
\vec{k}_{\perp}=\vec{p}_{q \perp}-\xi \vec{P}_{\perp}=-\vec{p}_{\bar{q} \perp}+(1-\xi) \vec{P}_{\perp}
\end{array}
$$

The subscript $\perp$ indicates the projection perpendicular to the spin quantization axis and the plus component of a 4 -vector $p \equiv\left(p^{0}, \mathbf{p}\right)$ is given by $p^{+}=p^{0}+\hat{\mathbf{n}} \cdot \mathbf{p}$; in eqs. 4) $\vec{P} \equiv\left(P^{+}, \vec{P}_{\perp}\right)=\vec{p}_{q}+\vec{p}_{\bar{q}}$ is the total momentum of the meson, and $\vec{p}_{q}\left(\vec{p}_{\bar{q}}\right)$ is the quark (antiquark) momentum. The structure of $M_{0}$ is more transparent if the fraction $\xi$ is replaced by the longitudinal momentum $k_{n}$ defined as

$$
k_{n}=\left(\xi-\frac{1}{2}\right) M_{0}+\frac{m_{\bar{q}}^{2}-m_{q}^{2}}{2 M_{0}}
$$


In terms of the new variable $\mathbf{k} \equiv\left(\vec{k}_{\perp}, k_{n}\right)$ the free mass operator is simply given by

$$
M_{0}=\sqrt{m_{q}^{2}+k^{2}}+\sqrt{m_{\bar{q}}^{2}+k^{2}}
$$

with $k^{2}=k_{\perp}^{2}+k_{n}^{2}$.

In this letter pseudoscalar S-wave mesons will be considered. Omitting for sake of simplicity the flavour and colour degrees of freedom, the intrinsic light-cone wave function has the following structure (cf. refs. [1, 2, 3, 4)

$$
\Psi\left(\vec{k}_{\perp}, \xi, \nu \bar{\nu}\right)=R\left(\vec{k}_{\perp}, \xi, \nu \bar{\nu}\right) \frac{w\left(k^{2}\right)}{\sqrt{4 \pi}} \sqrt{\frac{M_{0}}{4 \xi(1-\xi)}\left[1-\left(\frac{m_{q}^{2}-m_{\bar{q}}^{2}}{M_{0}^{2}}\right)^{2}\right]}
$$

where $\nu, \bar{\nu}= \pm 1 / 2$ are the spin projection variables and the momentum-dependent spin factor $R$ is

$$
R\left(\vec{k}_{\perp}, \xi, \nu \bar{\nu}\right)=\sum_{\nu^{\prime} \nu^{\prime}}\left\langle\nu\left|R_{M}^{\dagger}\left(\vec{k}_{\perp}, \xi, m_{q}\right)\right| \nu^{\prime}\right\rangle\left\langle\bar{\nu}\left|R_{M}^{\dagger}\left(-\vec{k}_{\perp}, 1-\xi, m_{\bar{q}}\right)\right| \overline{\nu^{\prime}}\right\rangle\left\langle\frac{1}{2} \nu^{\prime} \frac{1}{2} \overline{\nu^{\prime}} \mid 00\right\rangle
$$

In eq. (8) the $2 \times 2$ irreducible representation of the Melosh rotation [10] reads as follows

$$
\left\langle\nu^{\prime}\left|R_{M}\left(\vec{k}_{\perp}, \xi, m\right)\right| \nu\right\rangle=\chi_{\nu^{\prime}}^{\dagger} \frac{m+\xi M_{0}-i \sigma \cdot(\hat{\mathbf{n}} \times \mathbf{k})}{\sqrt{\left(m+\xi M_{0}\right)^{2}+k_{\perp}^{2}}} \chi_{\nu}
$$

where $\chi_{\nu}$ is the usual two-component Pauli spinor. The light-cone wave function (7) satisfies the following normalization condition

$$
\int d \vec{k}_{\perp} d \xi \sum_{\nu \bar{\nu}} \Psi^{\dagger}\left(\vec{k}_{\perp}, \xi, \nu \bar{\nu}\right) \Psi\left(\vec{k}_{\perp}, \xi, \nu \bar{\nu}\right)=\int_{0}^{\infty} d k k^{2}\left|w\left(k^{2}\right)\right|^{2}=1
$$

Following the Brodsky-Huang-Lepage prescription [1], the radial wave function $w\left(k^{2}\right)$ appearing in eq. (7) is identified with the equal-time wave function in the meson rest-frame. In this work we will adopt the effective $q \bar{q}$ Hamiltonian proposed by Godfrey and Isgur (GI) [6] for the description of meson mass spectra, viz.

$$
\begin{aligned}
H_{q \bar{q}} w\left(k^{2}\right)|00\rangle & \equiv\left[\sqrt{m_{q}^{2}+k^{2}}+\sqrt{m_{\bar{q}}^{2}+k^{2}}+V_{q \bar{q}}\right] w\left(k^{2}\right)|00\rangle \\
& =M_{q \bar{q}} w\left(k^{2}\right)|00\rangle
\end{aligned}
$$

where $M_{q \bar{q}}$ is the mass of the meson, $|00\rangle=\sum_{\nu \bar{\nu}}\left\langle\frac{1}{2} \nu \frac{1}{2} \bar{\nu} \mid 00\right\rangle \chi_{\nu} \chi_{\bar{\nu}}$ is the equal-time spin factor and $V_{q \bar{q}}$ is the effective $q \bar{q}$ potential. The interaction in the GI scheme, $V_{(G I)}$, is composed by a one-gluon-exchange term (dominant at short separations) and a flavour-independent linear-confining term (dominant at large separations). In order to analyze the effects of different terms of the GI $q \bar{q}$ interaction, two other choices of $w\left(k^{2}\right)$ will be considered; the first one is the solution of eq. (11) obtained after switching off the one-gluon-exchange part 
of $V_{(G I)}$, i.e., by retaining only its confining part, $V_{(\text {conf })}$, whereas the second choice is given by the solution of eq. (11) in which only the spin-independent part, $V_{(s i)}$, of $V_{(G I)}$ is retained. The three different forms of $w\left(k^{2}\right)$ will be denoted hereafter by $w_{(G I)}, w_{(\text {conf })}$ and $w_{(s i)}$, corresponding to $V_{(G I)}, V_{(c o n f)}$ and $V_{(s i)}$, respectively. The wave equation (11) is solved by expanding the wave function $w\left(k^{2}\right)$ onto a (truncated) set of harmonic oscillator (HO) basis states, viz.

$$
w\left(k^{2}\right)=\sum_{n=0}^{Q_{\max }} b_{n} R_{n, 0}\left(k^{2}\right)
$$

where $R_{n, \ell}$ is the usual HO radial function, $(n, \ell)$ are the HO quantum numbers and $Q_{\max }$ is the maximum number of $\mathrm{HO}$ excitation quanta included in the expansion (12). The coefficients $b_{n}$ are linear variational parameters, which are determined by applying to the Hamiltonian $H_{q \bar{q}}$ the Rayleigh-Ritz variational principle within the ansatz (12). We have checked that the value $Q_{\max }=38$ ensures a complete convergence for all the quantities considered in this work. As in ref. [6], the values $m_{u}=m_{d}=0.220 \mathrm{GeV}, m_{s}=0.419 \mathrm{GeV}$, $m_{c}=1.628 \mathrm{GeV}$ and $m_{b}=4.977 \mathrm{GeV}$ are adopted. We have also checked that, using the full GI $V_{q \bar{q}}$ interaction, the calculated mass spectra of pseudoscalar mesons coincide with the results of ref. [6]. The three wave functions $w_{(G I)}, w_{(\text {conf })}$ and $w_{(s i)}$ for the pion are compared in fig. 1: it can be seen that $w_{(G I)}$ is characterized by high momentum components $(k>0.5 \mathrm{GeV} / \mathrm{c})$ which are totally absent in $w_{(\text {conf })}$, whereas the effects of the spin-spin part of the one-gluon-exchange interaction strongly modifies the wave function at $k>1 \mathrm{GeV} / \mathrm{c}$. It should be pointed out that $w_{(\text {conf })}$ is similar to the gaussian-like wave function adopted in refs. [1, 2, 3, 国. The wave functions $w_{(G I)}$ of various pseudoscalar mesons are reported in fig. 2, where it can be seen that the high momentum tail of the wave function is similar in light and heavy pseudoscalar mesons. To sum up, the short-range structure of the effective $q \bar{q}$ interaction, determining the hyperfine splitting of hadron mass spectra, sharply affects the high momentum components of meson wave functions, i.e. it originates the presence of hard constituent quarks in mesons.

2. Meson electroweak properties. Let us now consider the charge form factor $F^{P S}\left(Q^{2}\right)$ and the axial-vector weak decay constant $f_{A}^{P S}$ of pseudoscalar mesons. As for the latter quantity, following the light-cone approach of ref. [4] and introducing an axial-vector coupling constant at the level of constituent quarks, $g_{A}^{q}(0)[2 \mathrm{~b}]$, one gets

$$
\begin{aligned}
f_{A}^{P S}= & g_{A}^{q}(0) \frac{\sqrt{6}}{(2 \pi)^{3 / 2}} \int d \vec{k}_{\perp} d \xi \sqrt{\frac{M_{0}}{4 \xi(1-\xi)}\left[1-\left(\frac{m_{q}^{2}-m_{\bar{q}}^{2}}{M_{0}^{2}}\right)^{2}\right]} \\
& \frac{w\left(k^{2}\right)}{\sqrt{4 \pi}} \frac{(1-\xi) m_{q}+\xi m_{\bar{q}}}{\sqrt{\xi(1-\xi)} \sqrt{M_{0}^{2}-\left(m_{q}-m_{\bar{q}}\right)^{2}}}
\end{aligned}
$$

The results of the calculations of $f_{A}^{P S}$ performed using $w_{(G I)}$ and $w_{(\text {conf })}$ in eq. (13), are reported in table 1 and compared with existing experimental data 112, 13 and lattice QCD calculations [14, 15, 16]. It can be seen that: i) $f_{A}^{P S}$ increases in presence of hard constituent 
quarks; ii) if $g_{A}^{q}(0)=1$ is used (as for the current quarks), the results obtained by considering only soft constituent quarks (i.e., by retaining only the confining term of the GI $q \bar{q}$ interaction) account for the data on $\pi$ and $K$ mesons, but they do not reproduce the lattice QCD predictions for heavier mesons; iii) in presence of hard constituent quarks (i.e., when the full GI $q \bar{q}$ interaction is taken into account), a reasonable agreement both with the data and the lattice QCD calculations can be obtained for all the considered pseudoscalar mesons by choosing $g_{A}^{q}(0) \neq 1$ and flavour independent (note that in table 1 the value $g_{A}^{q}(0)=0.726$ has been chosen in order to reproduce the central value of the experimental data for $\left.f_{A}^{\pi}\right)$. It should be pointed out that our predictions for the ratio $f_{A}^{P S} / f_{A}^{\pi}$, obtained using the full GI $q \bar{q}$ potential, compare favourably with the data and the lattice QCD calculations; this result depends only upon the GI wave function of the mesons, if $g_{A}^{q}(0)$ is assumed to be flavour independent. Furthermore, our results for the two ratios $f_{A}^{D_{s}} / f_{A}^{D}$ and $f_{A}^{B_{s}} / f_{A}^{B}$ are 1.17 and 1.20, respectively, in good agreement with the corresponding predictions $1.21 \pm 0.06$ and $1.22 \pm 0.02$ obtained from QCD sum rules [17.

Let us now investigate the sensitivity of the charge form factor of $\pi$ and $K$ mesons to soft and hard constituent quarks. As is known [1, 2, 3, 4, within the light-cone formalism the charge form factor is related to the matrix elements of the so-called good component of the electromagnetic current density, which is specified by its plus component $J^{+}$when the spin quantization axis is chosen so that the plus component of the four-momentum transfer vanishes $\left(Q^{+}=0\right)$. Following refs. [1, 2, 3, 4] and introducing a charge form factor of the constituent quark, $F^{q}\left(Q^{2}\right)[2 \mathrm{~b}]$, one gets

$$
F^{P S}\left(Q^{2}\right)=e_{q} F^{q}\left(Q^{2}\right) H\left(Q^{2}, m_{q}, m_{\bar{q}}\right)+e_{\bar{q}} F^{\bar{q}}\left(Q^{2}\right) H\left(Q^{2}, m_{\bar{q}}, m_{q}\right)
$$

where $e_{q}$ is the charge of the constituent quark and

$$
\begin{aligned}
H\left(Q^{2}, m_{1}, m_{2}\right)= & \int d \vec{k}_{\perp} d \xi \frac{\sqrt{M_{0} M_{0}^{\prime}}}{4 \xi(1-\xi)} \sqrt{\left[1-\left(\frac{m_{1}^{2}-m_{2}^{2}}{M_{0}^{2}}\right)^{2}\right]\left[1-\left(\frac{m_{1}^{2}-m_{2}^{2}}{M_{0}^{\prime 2}}\right)^{2}\right]} \\
& \frac{w\left(k^{2}\right) w^{*}\left(k^{\prime 2}\right)}{4 \pi} \mathcal{M}\left(\vec{k}_{\perp}, \vec{k}_{\perp}^{\prime}, \xi, m_{1}, m_{2}\right)
\end{aligned}
$$

with

$$
\begin{array}{r}
{\overrightarrow{k_{\perp}^{\prime}}}_{\perp} \vec{k}_{\perp}+(1-\xi) \vec{Q}_{\perp} \\
M_{0}^{\prime 2}=\frac{{k_{\perp}^{\prime 2}}_{m_{1}}^{2}}{\xi}+\frac{k_{\perp}^{\prime 2}+m_{2}^{2}}{(1-\xi)} \\
k_{n}^{\prime}=\left(\xi-\frac{1}{2}\right) M_{0}^{\prime}+\frac{m_{2}^{2}-m_{1}^{2}}{2 M_{0}^{\prime}}
\end{array}
$$

In eq. (15) $\mathcal{M}$ is the contribution of the Melosh rotations, which reads as

$$
\mathcal{M}\left(\vec{k}_{\perp},{\overrightarrow{k^{\prime}}}_{\perp}, \xi, m_{1}, m_{2}\right)=\frac{\xi(1-\xi)\left[M_{0}^{2}-\left(m_{1}-m_{2}\right)^{2}\right]+\vec{k}_{\perp} \cdot\left(\vec{k}_{\perp}^{\prime}-\vec{k}_{\perp}\right)}{\xi(1-\xi) \sqrt{M_{0}^{2}-\left(m_{1}-m_{2}\right)^{2}} \sqrt{M_{0}^{\prime 2}-\left(m_{1}-m_{2}\right)^{2}}}
$$


The results of the calculations of $F^{\pi}$ (eq. (14)), performed using the wave functions $w_{(G I)}$, $w_{\text {(conf) }}$ and $w_{(s i)}$ and $F^{q}=1$ (as for the current quarks), are shown in figs. 3 and 4 and compared with existing experimental data [18, 19, 20] at low and high values of $Q^{2}$. It can be seen that: i) the pion form factor is strongly affected by hard constituent quarks both at low and high $Q^{2}$; ii) the theoretical predictions based on soft constituent quarks only $\left(w_{(\text {conf })}\right)$, compare favourably with the experimental data for values of $Q^{2}$ up to several $(\mathrm{GeV} / \mathrm{c})^{2}$, in agreement with the results of refs. 11, 2, 3, 41. From fig. 3 it can also be seen that the charge radius $<r^{2}>_{\pi} \equiv-6\left(d F^{\pi} / d Q^{2}\right)_{Q^{2}=0}$ of the pion is strongly underestimated using the full GI $q \bar{q}$ potential. The agreement with the experimental value $\left(\left\langle r^{2}\right\rangle_{\pi}^{e x p}=(0.660 \pm 0.024 \mathrm{fm})^{2}\right.$ [20b]) can be recovered by considering $F^{q} \neq 1$ in eq. (14). Let us assume a simple monopole behaviour of the constituent quark form factor, viz.

$$
F^{q}\left(Q^{2}\right)=\frac{1}{1+Q^{2}<r^{2}>_{q} / 6}
$$

and fix $<r^{2}>_{u}=<r^{2}>_{d}=(0.48 \mathrm{fm})^{2}$ in order to reproduce the experimental value of the pion charge radius. The pion form factor obtained using in eq. (14) $F^{u}=F^{d}$ given by eq. (17) with $<r^{2}>_{q}=(0.48 \mathrm{fm})^{2}$, is represented by the solid line in figs. 3 and 4 . It can clearly be seen that the introduction of a single phenomenological form factor of the constituent quark is able to bring theoretical predictions, based on the full GI $q \bar{q}$ interaction, in agreement with data for values of $Q^{2}$ up to several $(G e V / c)^{2}$. The results for the kaon charge form factor are reported in fig. 5 and compared with existing experimental data [21, 22]. As in the case of the pion, we have considered both $F^{q}=1$ and $F^{q} \neq 1$; in the latter case a flavour independent form factor of the constituent quark, i.e. $F^{u}=F^{d}=F^{s}$, has been adopted using the monopole form of eq. (17) with $<r^{2}>_{q}=(0.48 \mathrm{fm})^{2}$ (i.e., the same charge radius of the constituent quark used for the pion). When $F^{q} \neq 1$ one gets $<r^{2}>_{K^{+}}=0.41 \mathrm{fm}^{2}$ and $<r^{2}>_{K^{0}}=-0.036 \mathrm{fm}^{2}$ to be compared with the experimental values $\left\langle r^{2}>_{K^{+}}^{\exp }=0.34 \pm 0.05 \mathrm{fm}^{2}\right.$ and $\left\langle r^{2}>_{K^{0}}^{\text {exp }}=-0.054 \pm 0.026 \mathrm{fm}^{2}\right.$ [21]. We would like to stress that the charge radius of the neutral kaon is independent of the charge radius of the constituent quark, if the latter is assumed to be flavour independent; thus, the agreement of our result of $\left\langle r^{2}>_{K^{0}}\right.$ with the data depends only upon the GI wave function of the kaon. The slight disagreement among the experimental and the calculated values of $\left\langle r^{2}>_{K^{+}}\right.$ might be ascribed to small deviations of $F^{s}$ from the $S U(3)$ symmetric value $F^{u}=F^{d}=F^{s}$.

In conclusion we have analyzed the wave function of pseudoscalar mesons within the relativized constituent quark model of ref. [6]. The large amount of high momentum components of the wave function, related to the one-gluon-exchange part of the effective $q \bar{q}$ potential, sharply affects the calculations of physical quantities, like the weak decay constant and the charge form factor, performed within the framework of the light-cone formalism. In particular, the ratio of the weak decay constant of flavoured pseudoscalar mesons to that of the pion is in agreement with the existing experimental data and the predictions of the fundamental theory; this fact gives us confidence in the overall behaviour of the GodfreyIsgur wave functions. The extension of our calculations to the nucleon electroweak properties is in progress and should allow to check the validity of the introduction of an axial-vector 
weak coupling constant and a charge form factor at the level of the constituent quark. As for the meson sector, the comparison of theoretical predictions with precise experimental data in a wide range of $Q^{2}$ for charge as well as transition form factors (as planned at CEBAF) should provide unique information on the $q \bar{q}$ component of meson wave functions.

We gratefully acknowledge T. Frederico and M. Strikman for enlightening discussions.

\section{References}

[1] P.L. Chung, F. Coester and W.N. Polyzou: Phys. Lett. B205 (1988) 545.

[2] a) Z. Dziembowsky: Phys. Rev. D37 (1988) 778; b) P.L. Chung and F. Coester: Phys. Rev. D44 (1991) 229.

[3] C.-R. Ji and S.R. Cotanch: Phys. Rev. D41 (1990) 2319.

[4] W. Jaus: Phys. Rev. D41 (1990) 3394; W. Jaus and D. Wyler: Phys. Rev. D41 1990) 3405; W. Jaus: Phys. Rev. D44 (1991) 2851.

[5] T. Frederico and G.A. Miller: Phys. Rev. D45 (1992) 4207.

[6] S. Godfrey and N. Isgur: Phys. Rev. D32 (1985) 185.

[7] S. Capstik and N. Isgur: Phys. Rev. D39 (1986) 2809.

[8] M.V. Terent'ev: Sov. J. Nucl. Phys. 24 (1976) 106; V.B. Berestetskii and M.V. Terent'ev. Sov. J. Nucl. Phys. 24 (1976) 547; ibid. 25 (1977) 347. For a review see B.D. Keister and W.N. Polyzou: Advances in Nuclear Physics, 20 (1991) 225.

[9] L.L. Frankfurt and M.I. Strikman: Phys. Rep. 76 (1981) 215.

[10] H.J. Melosh: Phys.Rev. D9 (1974) 1095.

[11] G.P. Lepage, S.J. Brodsky, T. Huang and P. Mackenzie : in Particles and Fields, edited by A.Z. Capri and A.N. Kamal (Plenum, New York, 1983), p. 83; S.J. Brodsky, T. Huang and G.P. Lepage, ibid., p. 143.

[12] B.R. Holstein: Phys. Lett. 244B (1990) 83.

[13] Particle Data Group, K. Hikasa et al.: Phys. Rev. D45 (1992) S1.

[14] UKQCD Collaboration: Nucl. Phys. B30 (Proc. Suppl.) (1993) 461. 
[15] C. Bernard et al.: Nucl. Phys. B30 (Proc. Suppl.) (1993) 465.

[16] A. Abada et al.: Nucl. Phys. B376 (1992) 172.

[17] C.A. Dominguez and N. Paver : Phys. Lett. B318 (1993) 629.

[18] C.N. Brown et al.: Phys. Rev. D8 (1973) 92.

[19] a) C.J. Bebek et al.: Phys. Rev. D9 (1974) 1229; b) C.J. Bebek et al.: Phys. Rev. D13 (1976) 25; c) C.J. Bebek et al.: Phys. Rev. D17 (1978) 1693.

[20] a) S.R. Amendolia et al.: Nucl. Phys. B277 (1986) 168; b) S.R. Amendolia et al.: Phys. Lett. 146B (1984) 116.

[21] S.R.Amendolia et al.: Phys. Lett. 178B (1986) 435.

[22] E.B. Dally et al.: Phys. Rev. Lett. 45 (1980) 232. 


\section{Table Caption}

Table 1. Axial-vector weak decay constant $f_{A}^{P S}$ (eq. (13)) calculated for various pseudoscalar mesons adopting different wave functions $w\left(k^{2}\right)$ (see text) and different values of the axial-vector weak coupling constant $g_{A}^{q}(0)$ for the constituent quarks. The results of the calculations are compared with existing experimental data [12, 13] and lattice QCD calculations [14, 15, 16]. 


\section{Figure Captions}

Fig. 1. The function $\left|k \cdot w\left(k^{2}\right)\right|^{2}$ for the pion, plotted versus the internal momentum $k$. Dot-dashed line: gaussian-like wave function of ref. [4]. Dotted line: wave function $w_{\text {(conf) }}$ obtained from eq. (11) using only the confinement part of the $q \bar{q}$ interaction of ref. [6]. Dashed line: wave function $w_{(s i)}$ solution of eq. (11) neglecting the spin-spin part of the $q \bar{q}$ interaction of ref. [6]. Solid line: wave function $w_{(G I)}$ obtained from eq. (11) using the full $q \bar{q}$ interaction of ref. [6].

Fig. 2. The function $\left|k \cdot w\left(k^{2}\right)\right|^{2}$ for various pseudoscalar mesons, obtained using in eq. (11) the full $q \bar{q}$ interaction of ref. [6]. Dot-dashed: $\pi$; dotted line: $K$; dashed line: $D$; dashed line with full dots: $D_{S}$; solid line: $B$; solid line with triangles: $B_{S}$.

Fig. 3. The square of the charge form factor of the pion (eq. (14)) at low values of $Q^{2}$. The dotted, dashed and dot-dashed lines represent the results of the calculations performed using the wave functions $w_{(\text {conf })}, w_{(s i)}$ and $w_{(G I)}$, respectively, and adopting $F^{q}=1$ (see text). The solid line is the result of the calculations of eq. (14) obtained using $w_{(G I)}$ and the monopole form for $F^{q}$ (eq. (17) with $<r^{2}>_{q}=\left(0.48 \mathrm{fm}^{2}\right)$. Experimental data are from refs. [18] (full dots) and [20] (open dots).

Fig. 4. The charge form factor of the pion (eq. (14)) versus $Q^{2}$. The dotted, dashed and dot-dashed lines represent the results of the calculations performed using the wave functions $w_{(\text {conf })}, w_{(s i)}$ and $w_{(G I)}$, respectively, and adopting $F^{q}=1$ (see text). The solid line is the result of the calculations of eq. (14) obtained using $w_{(G I)}$ and the monopole form for $F^{q}$ (eq. (17) with $<r^{2}>_{q}=\left(0.48 \mathrm{fm}^{2}\right)$. Experimental data are from refs. 18 (open dots), [19a] (full dots), [19b] (open squares) and [19c] (full squares).

Fig. 5. The same as in fig. 3, but for the kaon charge form factor. Experimental data are from refs. [21] (open dots) and [22] (full dots). 
TABLE 1

\begin{tabular}{|c|c|c|c|c|c|}
\hline & $\operatorname{con} f$ & $G I$ & $G I$ & \multirow[b]{2}{*}{ lattice $Q C D$} & \multirow[b]{2}{*}{ exp. data } \\
\hline meson & $g_{A}^{q}=1$ & $g_{A}^{q}=1$ & $g_{A}^{q}=0.726$ & & \\
\hline$\pi$ & 91.2 & 127.3 & 92.4 & & $92.4 \pm 0.2$ |12 \\
\hline $\bar{K}$ & 108.6 & 159.9 & 116.1 & $107 \pm 7 \quad$ |16 & $113.4 \pm 1.1$ |12 \\
\hline$D$ & 124.4 & 215.3 & 156.3 & \begin{tabular}{ll||}
$156 \pm 9$ & 14 \\
$147 \pm 8$ & $\mid 15$ \\
$154 \pm 7$ & 16
\end{tabular} & $<219$ 13 \\
\hline$\overline{D_{s}}$ & 142.1 & 252.8 & 183.5 & $\begin{array}{ll}163 \pm 7 & 15 \\
170 \pm 7 & 16\end{array}$ & \\
\hline $\bar{B}$ & 101.3 & 184.1 & 133.6 & \begin{tabular}{c|c||}
$141 \pm 16$ & 14 \\
$132 \pm 9$ & 15
\end{tabular} & \\
\hline$B_{s}$ & 116.8 & 220.6 & 160.1 & $146 \pm 7$ [15 & \\
\hline
\end{tabular}


This figure "fig1-1.png" is available in "png" format from: http://arxiv.org/ps/nucl-th/9405014v1 
This figure "fig2-1.png" is available in "png" format from: http://arxiv.org/ps/nucl-th/9405014v1 
This figure "fig1-2.png" is available in "png" format from: http://arxiv.org/ps/nucl-th/9405014v1 
This figure "fig2-2.png" is available in "png" format from: http://arxiv.org/ps/nucl-th/9405014v1 
This figure "fig1-3.png" is available in "png" format from: http://arxiv.org/ps/nucl-th/9405014v1 